\title{
Location as Interaction: Exploring Blended Spaces in the Global Village
}

\author{
Stephen Mokey \\ Rochester Institute of Technology \\ 152 Lomb Memorial Drive \\ Rochester, NY 14623, USA \\ swm6716@rit.edu
}

\author{
Alexander Nalbandian \\ Rochester Institute of Technology \\ 152 Lomb Memorial Drive \\ Rochester, NY 14623, USA \\ atn7080@rit.edu
}

\author{
Brian O'Keefe \\ Rochester Institute of Technology \\ 152 Lomb Memorial Drive \\ Rochester, NY 14623, USA \\ bjovks@rit.edu
}

\begin{abstract}
We present a novel means of experiencing an unfamiliar tourist destination using location-based interaction design with mobile devices. Tracking a visitor's location allows us to automatically present the visitor with relevant information as soon as they arrive at a point-of-interest. By synchronizing the delivery of digital content with the visitor's physical location, the visitor is able to move through and explore the physical and digital spaces simultaneously, using a locationbased interface to experience a blended space. We theorize that this location-based content delivery method will instill a sense of freedom and immersion as the visitor explores the blended space in a natural and serendipitous way. To test our theory and validate our design strategy, we have developed a prototype application called Global Village Explorations.
\end{abstract}

Tourism; mobile; blended spaces; user experience.

\section{INTRODUCTION}

Worldwide tourism is an immense industry, with 980 million individual tourist visits occurring in 2011, an increase of 4.4\% from 2010 (World tourism, 2012). However, even as the tourism industry grows, many public tourism venues in the United States are experiencing problems. The American National Parks have revealed that the current recession has led to budget cuts and reduced guest services in parks. However, there are many opportunities to supplement these lost resources through blended spaces that incorporate a visitor's mobile device into the experience. (Benyon 2013, Imaz \& Benyon, 2005) Our contribution is to leverage location tracking on mobile devices to design for blended user experiences while looking at how people visit univerity campuses.

This paper is the result of outcomes from the Mobile Experiences for Tourism, a Masters $\mathrm{HCl}$ seminar course at the Rochester Institute of Technology $(R I T)^{1}$. This course was created with the purpose of researching new methods to design pervasive mobile tourism experiences for university and historical visits. Our research has led to the design of the Brick City Tours, a mobile platform to entertain and personalize campus visits by

\footnotetext{
${ }^{1}$ http://wallacecenter.rit.edu/tls/students-creatingmobile-experiences
}

designing for the problems around selecting a university while on a campus tour (O'Keefe 2013).

This paper focuses on one aspect of the Brick City Tours, audio driven location-based interactions in the Global Village at RIT. A proof-of-concept prototype, Global Village Explorations (GVE), was developed to test our location-centered design strategy. Our prototype is available in RIT's Global Village, for convenience and to provide an outlet for further testing and development. RIT's admissions department has expressed interest in developing a similar app for prospective students, so we wanted to explore this concept in a small, self-contained section of the campus. This application design is very specific to the RIT Global Village, but the concepts explored are generalizable to other tourism research under development at $\mathrm{RIT}^{2}$. It is our goal to explore novel methods of interaction for use in tourism sites in the greater Rochester, NY area.

Our design strategy for blended spaces involves the de-emphasis of disruptive visual interaction in the digital space, to maintain an unbroken sense of the physical space and maximize freedom within that space. Existing systems focus on utility without considering the full breadth of the experience. Users of these systems may focus on the digital information to the detriment of experiencing their physical surroundings, a concept we have dubbed

\footnotetext{
${ }^{2}$ www.blendedInteractions.com
} 
"periscope tourism", i.e. living through the lens. (Benyon 2013) Several previous studies have explored methods of reducing the necessity of digital interruption (Holland, Morse, \& Gedenryd, 2002) (Robinson et al., 2010), (Strachan \& MurraySmith, 2009), (Strachan, Williamson, \& MurraySmith, 2002). The navigational aids in these studies refrain from using visual feedback to guide the user, which might constitute a disruptive transition of the user's attention between physical and digital spaces. Utilizing a non-visual method of accessing and presenting information may allow the design of blended spaces that successfully merge the physical and digital spaces into a cohesive user experience.

\subsection{Designing with Blends in the Global Village}

According to Benyon, the concept of a blended space has emerged relatively recently, as a result of designers grappling with the multitude of new design challenges presented by mixed reality (Imaz \& Benyon, 2005). By reducing the visibility of technology, we hope to create a more immersive, blended space: a space that unifies the physical and digital spaces. Rather than including a method of navigation wherein the digital space offers constant navigational feedback, navigation assistance in GVE is silent for the majority of the experience. Visitors are free to explore the Global Village and approach whatever interests them, with minimal interference from the digital space. When the visitor enters the vicinity of a POI, also known as a "hotspot", the mobile device notifies them that additional information is available. In addition to POI notifications, we gently warn the visitor if they leave the area encompassed by the blended space: when they cross the digital "fence". We call this blended space implementation "fenced hotspots". Our design uses the visitor's position in the tourism space as a life-size user interface, at a human scale (Imaz \& Benyon, 2005). The user's movement controls the information presented by the corresponding digital space: a location-based UI. The synchronization between physical and digital is intended to be seamless, so that the visitor is simultaneously interacting with both spaces through their deliberate movement within the blended space.

In the following sections, we present an overview of mixed reality tourism experiences through the lens of blended spaces, followed by an introduction to our design strategy and proof-of-concept prototype. Finally, we present the description and results of a qualitative usability test, which begins to validate our design strategy.

\section{RELATED WORK}

Several other studies have developed mixed reality tourism experiences to help visitors navigate through an unfamiliar environment and retrieve contextual information about POIs. From audio and haptic guidance (Holland, Morse, \& Gedenryd, 2002), (Robert \& Breazeal, 2012), (Strachan \& Murray-Smith, 2009), (Strachan, Williamson, \& Murray-Smith, 2002) to augmented reality (AR) (Schinke, Henze, \& Boll, 2010) and interactive puzzles (Cabrera et al., 2005) to digital tour guides (Rocchi et al., 2004), different designs have been utilized to enhance the visitor's experience in mixed reality environments. Some designs focus on utility, such as offering navigational guidance for traveling between POls (Holland, Morse, \& Gedenryd, 2002), (Strachan \& Murray-Smith, 2009), (Strachan, Williamson, \& Murray-Smith, 2002). Others generate dynamic paths and accompanying narratives based on POls encountered along the way (Schöning, Hecht, \& Starosielski, 2008). Still others avoid choosing a single path altogether, and instead give the user freedom to explore while still providing subtle feedback to help visitors stay on track (Robinson et al., 2010).

Most applications offering AR guidance rely on the destination POI being within the camera's current field of view. However, Schinke et al. (2010) have devised a novel method of showing off-screen POls using a set of arrows rendered into the scene, and have compared this method to a non-AR approach using a minimap. They theorized that the $A R$ design would show visitors nearby POls in a more intuitive way, since less cognitive effort is required for the visitor to mentally map his position relative to the POls being displayed. The experimental results lead the authors to conclude that their AR method of showing off-screen POIs was more effective than the minimap technique. An analysis of this comparison using blended theory would suggest that AR helps unify the physical and digital spaces, letting the user remain in the context of his surroundings while receiving information about nearby POls.

Rocchi et al. (2004) describe a system for helping a visitor explore a museum. As the visitor moves freely between exhibits, a virtual tour guide transfers seamlessly between mobile and installation displays to direct the visitor's attention and deliver information. The visitor is able to explore the museum in any way they choose and the mobile guide will follow them and provide information as they move between exhibits. Through the lens of blended spaces, we see that the physical and digital spaces remain highly synchronous; information from the digital space is automatically presented as the visitor's location changes. 
Cabrera et al. (2005) present an analysis of a mobile application that provides schoolchildren with interactive puzzles as they explore a museum. Visitors use information from museum exhibits to complete a series of challenges that involve rearranging images and text. Experimental results showed that participants became extremely focused on completing the digital puzzles, to the detriment of experiencing the exhibits themselves. Cabrera et al. note that visitors "used the exhibits ... as auxiliary material towards the main objective, i.e. solving the given [digital] problem" (Cabrera, 2005). From the perspective of blended spaces, we might say that the digital space drew visitors' attention away from the physical space, despite the explicit link between them. The physical space became simply a means to an end in the digital space because the relationship was unidirectional; the digital experience depended on information from the physical space, but the physical space had no connection back to the digital. A design that merges the two spaces in a bidirectional, symbiotic way might avoid this issue.

By analyzing these implementations of mixed reality through the lens of blended spaces, we hope to apply similar techniques in the creation of a blended space prototype. The next section introduces the prototype and gives insight into the design strategy behind our blended space.

\section{GLOBAL VILLAGE EXPLORATIONS}

The Global Village Explorations (GVE) prototype was created as a proof-of-concept prototype to test our approach to location-based interaction design. The physical space used in the prototype covers a small subsection of the RIT campus, the Global Village. The Global Village is a cluster of buildings surrounding an open quad approximately 9000 square meters in size. The area contains several buildings, shops, restaurants, and areas for eating and socializing, giving it a wealth of POls for visitors to explore. The presence of buildings along three out of four sides also gives the area a natural sense of bounded space that is ideal for digital fencing. Due to limitations in the accuracy of civilian GPS technology, not every location in the Global Village could be marked as a POI. This meant selecting locations that were at least 15 meters apart, so that geo-fences could be at least 10 meters in radius to accommodate a 7 meter uncertainty.

GVE features RIT's mascot, Ritchie the Tiger, whose character delivers audio POI information. When GVE is started, a photo of the Global Village is shown next to a large button labeled "Start Exploring" (figure 1). When the visitor presses this button, an image of Ritchie the Tiger is shown, accompanied by Ritchie introducing himself. Ritchie tells the visitor that he will notify them when he has more information to share. The visitor can then put the phone in standby mode and keep it in their pocket; the location tracking functionality has been started, and the visitor is free to start exploring the Global Village area. When the visitor reaches a $\mathrm{POI}$, Ritchie gives visitors a short description of the $\mathrm{POI}$ and some relevant details. For example, at the Cantina \& Grille, visitors are told about the different cuisines offered by each food counter and the availability of outdoor seating in warm weather. The Ritchie character's fun personality also comes through in his dialogue: his description of the Cantina \& Grille ends with his remarking that all this talk about food has made him hungry.

\subsection{Location-Based Interaction}

Six POls throughout the Global Village were chosen: the entrance, Innovation Centre building, Global Plaza, ShopOne2 gift shop, Market store, and Cantina \& Grille. Hotspot sizes for these POIs vary from 10 meters to 20 meters in radius. When a hotspot is entered, the mobile device vibrates and plays Ritchie's roar to notify the visitor that Ritchie has information about the POI. Visitors can then take out their device and press the Play button to hear what Ritchie has to say. Ritchie's descriptions of the visitor's surroundings, i.e. information from the digital space, are played aloud to the visitor as they remain visually immersed in the physical space. After the visitor has finished listening to Ritchie's information, they can continue exploring.
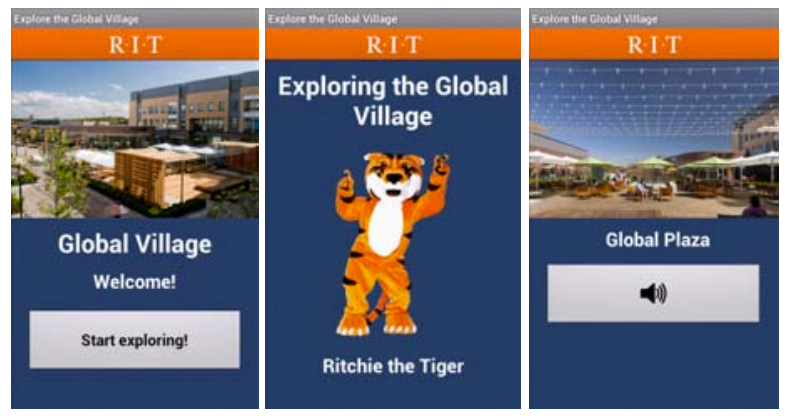

Figure 1: The GVE application's start, exploration, and hotspot screens

The use of location-based interaction is intended to augment the user experience in three ways. First, the mobile device remains in the visitor's pocket as they explore, allowing the user to experience the destination without distraction. Second, the digital space only presents information relevant to the visitor's current location and uses sound for content delivery, minimizing the need to interact directly with the device. Third, the lack of navigational guidance means the visitor can move through the area however they wish. Navigational cues are given only when visitors cross the digital "fence" (figure 2). By encouraging serendipitous 
exploration, we believe visitors will have a more exciting and engaging experience.

\subsection{Ritchie the Tiger}

An important aspect of the blended experience is the narrator, Ritchie the Tiger, whose voice and character appear in the prototype. While he does not physically accompany the visitor during their exploration, his narration provides a link between the digital and physical spaces. Although Ritchie's presence in the physical space is limited to his roars, voice, and vibrations, the decision not to provide on-screen text of Ritchie's spoken dialogue was intended to reduce the visitor's perception of Ritchie as a purely digital character. Robert and Breazeal (2012) show that children who interact with blended reality characters engage in play that is more imaginative and longer in duration. Their results suggest that blended characters are more engaging than those that are purely digital.

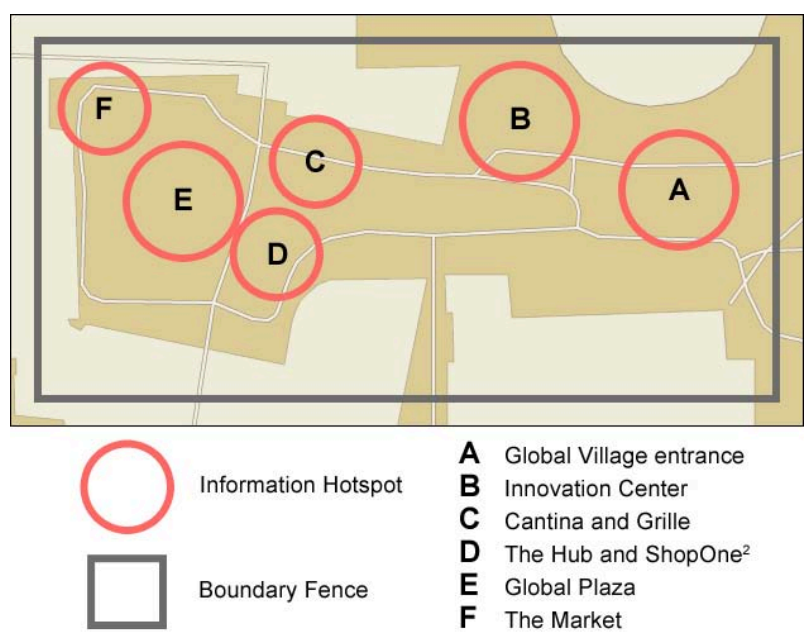

Figure 2: Global Village area with annotated information hotspots and boundary fence

\section{EVALUATION}

To begin validating the design strategy behind our prototype, we recruited ten groups of 2-4 participants for a usability test. Participants were high school students and their families visiting RIT as prospective applicants, which ensured that none of the participants had more than a passing familiarity with the Global Village area. Visitor recruitment was conducted by setting up a table near the Global Village during an RIT open house. We approached visitor groups directly with an invitation to participate.

Each group of participants was given a single Android mobile device with the GVE prototype installed and running. After giving each group a brief UI overview, they were led to the entrance of the Global Village. Participants were then told they were free to explore the area and interact with
Ritchie as appropriate. While the participants explored, an experimenter shadowed the group to record relevant information.

\subsection{Results}

Our preliminary usability test was intended to give our design team qualitative data regarding locationbased interactions in a blended space. Our testing was not intended to be deeply rigorous; rather, its purpose was to provide a starting point from which to further develop our design strategy.

Our location-based interaction design was rated satisfactory by seven of ten groups who enjoyed the freedom of exploring the space. However, three of ten groups would have preferred more guidance; one participant remarked that it "felt like walking in the dark". Due to the natural sense of bounding in the space, the digital fence rarely came into play. Participants described Ritchie's information as interesting and concise, though some participants anticipated that every building would have informational content.

The combination of the tiger's roar and vibration effect that were used for notification was well received by eight of ten participant groups. However, some individuals were either unable to sense the notification or thought using both sound and vibration was redundant. Several participants suggested that the ability to configure notification settings could improve the experience by providing more personalization.

The amount of guidance had mixed reviews, but most participants suggested increasing it. Allowing the user to customize the level of guidance was a suggestion offered by several participants. Levels of guidance could range from GVE's hands-off style to explicit navigational assistance for guiding users directly to a chosen POI. A compromise between these two solutions might be a list of POls. Unlike a map, a list could still minimize directional guidance, but would also enable users to review previously visited POls and see which POls they haven't yet visited.

\section{DISCUSSION}

In reviewing participants' feedback, we find data that supports our design strategy, in addition to opportunities for improvement. While a majority of participants responded positively to the concept of non-guided exploration, a significant number expressed the desire for more guidance. One possible explanation is that participants value different things in a mobile experience. Not all tourists will sympathize with our goal of reducing interaction with their mobile devices; some may prefer to exert personal control over information access and navigational guidance. Another 
possibility is that participants' motivations were not in line with those of our intended users. Our design targets tourists who are visiting a destination for the experience, while the participants in our study may not have had any base interest in visiting the Global Village.

Along with mixed feedback on guidance, participants provided a range of responses regarding the implementation of the Ritchie character. We believe that offering more customization options for delivery of digital content should please a wider spectrum of users. Also, improving the quality of information offered by the character, along with the writing, voice acting, and other aspects of production, could make the character more useful and believable to users.

Our next effort is centered on improving the fidelity of the graphical and media elements of the experience, along with the appropriateness of digital content. For further studies in location-based interaction and blended space design, we are currently working on the grant-funded Mobile Experiences for Tourism Project (O'Keefe, Benyon 2013). The Mobile Experiences for Tourism Project is made possible by the New York State Council on the Arts with the support of Governor Andrew Cuomo and the New York State Legislature ${ }^{3}$.

\section{CONCLUSION}

The concept of a blended space offers a simple but powerful way to develop new mixed reality experiences for a given location. Markers such as QR codes can get in the way of a great blended experience, but mobile features like locationtracking, RFID, and Near-Field Communication (NFC) will increasingly replace them. Rather than asking the user to step out of the flow of the whole experience in order to align the phone and code, these technologies provide a more seamless touch point between the physical and the digital to provide a better, blended UX.

The design and testing of the blended experience provided by the Global Village Explorations application yielded qualitative data that will be valuable in our on going research in the tourism domain. We continue to collaborate with stakeholders in the State of New York and the GCVM as we develop immersive mobile user experiences for the NYS tourism locations. We consider the GVE prototype to be a successful first step in the development of our design strategies and user experience goals.

\section{REFERENCES}

\footnotetext{
${ }^{3}$ http://blendedinteractions.com/projects/nysca-mobiletourism/
}

Benyon, D., Quigley, A., O’Keefe, B., \& Riva, G. "Presence and Digital Tourism"Al \& Society. 10.1007: (2013).

Cabrera, J. S., Frutos, H. M., Stoica, A. G., Avouris, N., Dimitriadis, Y., Fiotakis, G., and Liveri, K. D. Mystery in the museum: collaborative learning activities using handheld devices. In Proc. MobileHCI 2005. ACM Press (2005), 315-318.

Holland S., Morse D. R., and Gedenryd, H. AudioGPS: Spatial Audio Navigation with a Minimal Attention Interface. Personal Ubiquitous Comput. 6, 4 (January 2002), 253-259.

Imaz M. and Benyon, D. Designing with Blends. MIT Press (2005).

O'Keefe, B., Slutsky, B., Iuliucci, N., Nalbandian, A., Thanedar, A., Mokey, S., \& Mival, O. Mobile Experiences for Tourism: Brick City Tours. CHI 2013 Work-in-Progress. ACM Press 2013.

O'Keefe, B., Benyon, D. \& Mival, O. A Blended Space for Tourism: Genesee Country Village \& Museum. CHI 2013 Blend13 Workshop. ACM Press 2013.

Robert, D. and Breazeal, C. Blended reality characters. In Proc. HRI 2012. ACM Press (2012), 359-366.

Robinson, S., Jones, M., Eslambolchilar, P., Murray-Smith, R., and Lindborg, M. "I did it my way": moving away from the tyranny of turn-byturn pedestrian navigation. In Proc. MobileHCl 2010. ACM Press (2010), 341-344.

Rocchi, C., Stock O., Zancanaro, M., Kruppa, M., and Krüger, A. The museum visit: generating seamless personalized presentations on multiple devices. In Proc. IUI 2004. ACM Press (2004), 316-318.

Schinke T., Henze, N., and Boll, S. Visualization of off-screen objects in mobile augmented reality. In Proc. MobileCHI 2010. ACM Press (2010), 313-316.

Schöning, J., Hecht, B., and Starosielski, N. Evaluating automatically generated locationbased stories for tourists. In Proc. CHI 2008. ACM Press (2008), 2937-2942.

Strachan, S. and Murray-Smith, R. Bearing-based selection in mobile spatial interaction. In Personal Ubiquitous Comput. 13, 4 (May 2009), 265-280.

Strachan S., Williamson J., and Murray-Smith, R. Show me the way to Monte Carlo: density-based trajectory navigation. In Proc. CHI 2007. ACM Press (2002), 1245-1248.

World tourism barometer excerpt. Volume 10, March 2012. UNWTO (2012), 1-5. 